\title{
Task-irrelevant visual motion and flicker attenuate the attentional blink
}

\author{
ISABEL AREND, STEPHEN JOHNSTON, and KIMRON SHAPIRO \\ University of Wales, Bangor, Wales
}

\begin{abstract}
Our reduced ability to correctly report two sequentially presented targets is seen in the robust effect known as the attentional blink (AB; Raymond, Shapiro, \& Arnell, 1992). One recent report (Olivers \& Nieuwenhuis, 2005) strikingly reveals the AB to be virtually abolished when non-task-demanding music occurs in the background. The authors suggest that a diffuse attentional state is the mediating factor. Here, we seek to broaden the finding's generality by determining if task-irrelevant visual motion and flicker also attenuate the AB. In our experiments, the AB task was presented together with a background field of moving dots that could move away from or toward the central AB task, or flicker. In the control condition, the dots remained static. The AB was attenuated-though to different degrees-in all experimental conditions, but not in the static condition. Our findings add to the generality of the previous conclusions, and we emphasize an account based on the overallocation of attention.
\end{abstract}

Humans reveal an important constraint on attention when asked to respond to two events presented within a short temporal interval. This constraint is clearly illustrated by the phenomenon referred to as the attentional blink (AB; Raymond, Shapiro, \& Arnell, 1992). In a typical AB experiment, observers are asked to report two targets (T1 and T2) embedded in a temporally displayed stream of nontargets (i.e., distractors). The temporal interval between the two targets varies across trials. The fundamental outcome is that T2 report accuracy decreases when it appears within a $100-500-\mathrm{msec}$ interval after T1. This phenomenon has been replicated frequently using various types of stimuli, including letters (Raymond et al., 1992), colors (Ross \& Jolicœur, 1999), orientation (Joseph, Chun, \& Nakayama, 1997), and words (Olson, Chun, \& Anderson, 2001).

Theoretical accounts of this effect are based on the idea that limited attentional resources must be deployed to process $\mathrm{T} 1$. As a result, the attentional mechanisms required to fully process T2 become temporarily unavailable, which impedes T2 from reaching the higher level of processing necessary for conscious report. However, when the temporal interval between the two targets is extended (500-700 $\mathrm{msec}$ ), attentional resources become available and can be used to process T2 (Chun \& Potter, 1995; Giesbrecht \& Di Lollo, 1998; Raymond et al., 1992; Shapiro, Arnell, \& Raymond, 1997).

Despite the hundreds of published reports underscoring the robustness of the $\mathrm{AB}$ outcome, a few conditions have emerged in which an $\mathrm{AB}$ does not occur, even though the

\footnotetext{
We gratefully acknowledge Wellcome Trust Project Grant 071944, which provided funding for the second and third authors. Correspondence concerning this article should be addressed to I. Arend or K. Shapiro, Wolfson Centre for Cognitive Neuroscience, School of Psychology, Brigantia Building, University of Wales, Bangor, Gwynedd LL57 2AS, Wales (e-mail: i.arend@bangor.ac.uk, k.shapiro@bangor.ac.uk).
}

necessary prerequisites have been met (cf. Shapiro, Raymond, \& Arnell, 1997). A recent example from Kellie and Shapiro (2004) used morphing technology to allow target and distractor stimuli to be changed gradually from one object to another. The outcome was that the AB was present when $\mathrm{T} 1$ and $\mathrm{T} 2$ were selected from a rapid stream of visually presented items (RSVP) containing nonmorphing stimuli, but was significantly attenuated when targets were selected from a stream containing morphing stimuli. This result was interpreted to suggest that the formation of a single object file, linking $\mathrm{T} 1$ to $\mathrm{T} 2$, prevents the AB from occurring. Sheppard, Duncan, Shapiro, and Hillstrom (2002) also found that the AB was absent when the first target required a gap-duration judgment ${ }^{1}$ (Experiment 1), as well as when it required judging the duration of a letter (Experiment 2). However, when the "single-stream" percept was broken down by the inclusion of a longer gap, the $\mathrm{AB}$ was again observed (Experiment 4). In a different context, Ogawa and Suzuki (2004) found that the AB was absent when T2 was a negative stimulus, ${ }^{2}$ suggesting that emotional valence of the second target is an important factor in determining whether or not a given stimulus reaches awareness. In a similar fashion, Shapiro, Caldwell, and Sorensen (1997) showed that participants searching for their own name, as T2, did not experience an AB. Taken together, these results show that the $A B$ is a robust phenomenon in most experimental situations, with very few exceptions. Such exceptions, however, should not be overlooked and instead must be viewed as providing a critical window into the mechanism of divided-attention tasks such as the AB. Recently, a new example of the failure to obtain an AB has emerged (Olivers \& Nieuwenhuis, 2005), and its implications for understanding the limits of visual attention are the basis of the present exploration.

In their study, Olivers and Nieuwenhuis (2005) revealed the beneficial effect of secondary task-irrelevant activity on the AB. Their study was motivated by the observa- 
tion that people could better perform the $\mathrm{AB}$ task when they were distracted by various means from the task. In their study, one (control) group of participants performed a typical $\mathrm{AB}$ task with parameters similar to those used in previously reported studies. Another (experimental) group was asked to perform the same $\mathrm{AB}$ task, with the only change being that they also listened to music during the experimental session. The surprising outcome of this study was that the $\mathrm{AB}$ was absent for the group that listened to music. Such an outcome also manifested itself for another condition, in which participants free associated while performing the task but the $\mathrm{AB}$ did not occur when they were rewarded for good performance. The "blink" for the first group was characterized by the often-found Ushape function, with a magnitude similar to that reported in previous $\mathrm{AB}$ studies. The authors interpret this finding to argue that a secondary, irrelevant task induces a diffuse attentional state, suggesting that the arousal and/or the affective state of the observer may change in some unspecified way to facilitate performance.

Olivers and Nieuwenhuis's (2005) supposition that a diffuse attentional state might serve to benefit an attentionally demanding task such as the $\mathrm{AB}$ is not unsupported by the literature. Snodgrass, Shevrin, and Kopka (1993) found that highly motivated participants actually performed worse on a subliminal perception task when allowed to use a preferred strategy. They argued that less, rather than greater motivation - that is, relaxation, in the context of the present report-facilitated performance on an attentionally difficult task. More recently, Smilek, Enns, Eastwood, and Merikle (in press) similarly found that performance in a difficult visual search task was improved by instructing participants to relax. The theoretical question that motivated the present research was an attempt to understand the mechanism by which relaxation improved performance on an attentionally demanding task such as the AB.

In seeking an account, we hypothesized that the $\mathrm{AB}$ task might elicit an overcommitment of attentional resources to the first target, over and above that required to perform the task to the criterion set by experimental instruction. Following logically from this, anything that prevents overallocation of attention should in turn leave more resources for the second target task. We chose a novel way to test this hypothesis by manipulating the visual background in a manner designed to decrease spatial attention to the location of the AB task. Such a manipulation enabled us, moreover, to determine if the observed benefit of the secondary task-irrelevant activity is restricted to experimental situations in which the distracting task is presented in a modality different from that required by the AB task. More specifically, we sought to determine whether taskirrelevant visual motion would attenuate the $\mathrm{AB}$ outcome and subsequently, whether the direction of motion would exert a differential effect. To anticipate our findings, background motion away from the primary $\mathrm{AB}$ task virtually fully attenuates the $\mathrm{AB}$ effect, whereas motion toward the primary task and flicker have an intermediate effect.

\section{EXPERIMENT 1}

In our first experiment, we sought to study whether an irrelevant visual background would benefit participants' performance in a visual $\mathrm{AB}$ task, as did the multisensory music in the Olivers and Nieuwenhuis (2005) study. Our strategy was to create an experimental situation in which changes in the visual field happened in parallel with the AB task. We anticipated that changes in the visual field produced by the background would draw spatial attention away from the RSVP stream, thereby reducing the AB effect. We implemented this by adding stimuli in motion at the perimeter of the RSVP stream. We chose a starfield simulation technique that creates an apparent motion illusion of dots moving, in the depth plane, away from the center of the screen, where the AB task occurred. In this first study, we compared participants' performance in such an $\mathrm{AB}$ task (out) to a control condition in which the same round dots appeared stationary (static).

\section{Method}

Participants. Twenty-two participants were recruited from the community panel, and each received $£ 5$ to take part in the present study. The participants were allocated randomly to one of the two conditions, resulting in 11 participants per condition: The participants' mean age in the out condition was 28 years $(S D=5.41)$; it was 27 years $(S D=4.9)$ in the static condition. All of the participants reported normal or corrected-to-normal visual acuity.

Stimuli and Procedure. In the AB task, the target stimuli were white digits between 2 and 9, inclusive, and the distractors were uppercase white letters, excluding I, O, Q, and S, presented on a black background. Stimuli were presented in the center of a screen with a resolution of $1,024 \times 768$, in a 50-point Arial font. All items in the stream were presented for $64 \mathrm{msec}$ with a 16 -msec ISI, yielding a stimulus presentation rate of 13.5 items per sec.

The experiment was programmed using the MATLAB software package with the Psychophysics Toolbox extensions (Brainard, 1997; Pelli, 1997). The trials started with a center fixation dot with starfield surround for $800 \mathrm{msec}$, for both the static and motion conditions. After this period, the RSVP stream, containing 18 items including the two targets, was shown for $1.44 \mathrm{sec}$. While the RSVP stream was shown, the starfield continued to run. A $100 \times 100$ pixel area centered on the RSVP stream prevented "mixing" of the RSVP stream and the starfield - that is, the starfield stopped short of this area. At the end of the RSVP stream, a fixation dot was shown while the starfield continued for an additional $1.76 \mathrm{sec}$. T1 could appear at position 3,5 , or 7 , relative to the beginning of the stream; T2 could appear at either position $2,3,6$, or 8 after T1.

The starfields were constructed of 32 particles; each particle was a round dot varying in diameter, randomly, between 1 and 10 pixels. The moving starfield showed particles traveling from the center of the screen to the periphery, creating an apparent outward motion (see Figure 1, top panel); the static starfield was created by randomly choosing one frame from the motion set. Dots disappearing from the edge were replaced by new dots appearing from their "origin" near the RSVP stream. This frame remained constant throughout the static trials (see Figure 1, bottom panel). We will always refer to our moving starfield as out and the nonmoving one as static.

The participants were asked to read the instructions, which clearly stated that it was important to identify the only two digits in the stream and to ignore the particles that would appear around the stream. At the beginning of each trial, a message appeared on the screen indicating that the participants had to press the mouse button to start the trial. At the end of each trial, the participants were 

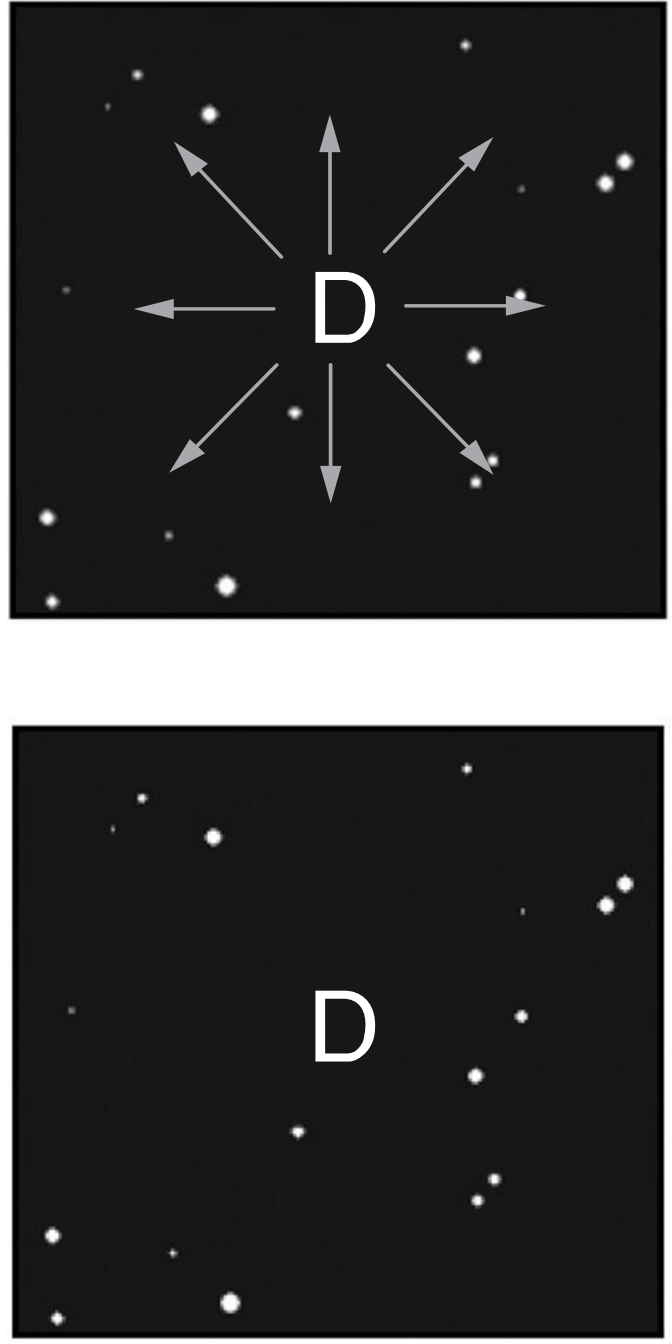

Figure 1. The top panel shows the out motion condition from Experiment 1. The round dots from the starfield move from the center of the screen to the periphery. The bottom panel shows an example of the static condition.

asked to type their responses by using the computer keyboard. They were encouraged to guess, when uncertain what the targets were. All of the participants received 12 practice trials before beginning the experimental block. The experimental block consisted of 21 trials per each SOA level, resulting in 84 trials. The entire session took approximately $45 \mathrm{~min}$.

Design. This experiment employed a $4 \times 2$ mixed factorial design. The within-group factor (lag) was the temporal interval between T1 and T2, which had four levels, 2, 3, 6, and 8, corresponding to the appearance of T2-160, 240, 400, and $560 \mathrm{msec}$ after T1. The between-groups factor ( field) was the direction of motion of the starfield, which had two levels: out and static.

\section{Results and Discussion}

Overall T1 accuracy was $.90(S D=.07)$ for the out condition and $.90(S D=.04)$ for the static condition. Estimates of second target identification were based solely on trials in which the first target had been reported correctly. This way of quantifying performance in $\mathrm{AB}$ experiments is frequently adopted, because on trials in which the first target is not correctly identified, the source of the error is unknown; in such cases, the attentional effects on secondtarget processing cannot be estimated. Figure 2 shows the mean proportion of T2 accuracy in trials in which T1 was also reported correctly for both static and out conditions as a function of lag.

A two-way mixed ANOVA for field (2) and lag (4) yields a significant main effect of lag $[F(3,60)=$ $\left.11.743, p<.05, \eta_{\mathrm{p}}^{2}=.370\right]$ and a significant main effect of field $\left[F(1,20)=5.297, p<.05, \eta_{\mathrm{p}}^{2}=.209\right]$, as well as a significant interaction between field and lag $[F(3,60)=$ $\left.4.201, p<.05, \eta_{\mathrm{p}}^{2}=.174\right]$. Pairwise comparisons using Fisher's test revealed that the two groups differ at lag 2 $(p<.05)$ and lag $3(p<.05)$, but not at lag 6 and lag 8 . One-way ANOVA tests were conducted separately for each one of the background conditions. The effect of lag reached significance for the static condition $[F(3,30)=$ $\left.10.075, p<.05, \eta_{\mathrm{p}}^{2}=.502\right]$ but not for the out condition $(F<1)$, clearly revealing that an AB occurred for the former but not for the latter condition. These results demonstrate that an irrelevant visual background attenuates the $\mathrm{AB}$.

As in the study performed by Olivers and Nieuwenhuis (2005), our results reveal a dramatic reduction in the dual-task cost typical of AB experiments of this nature. Moreover, because the background in the present experiment was delivered in the same modality as the primary AB task, we show that the background does not have to be from a different modality and that a visual modality background acts in the same manner as one from the auditory modality. Furthermore, we have shown that a very

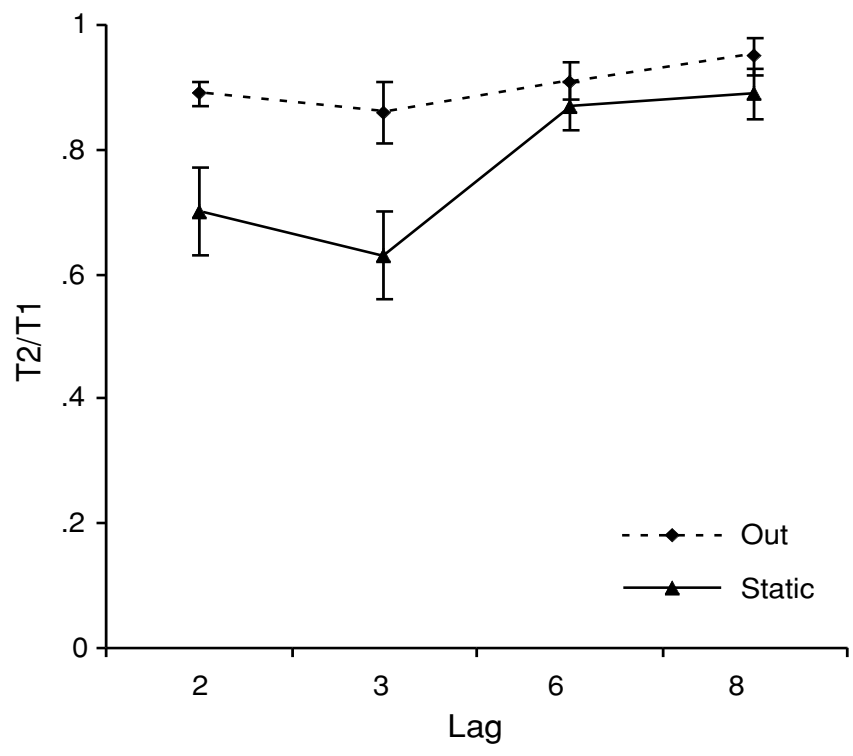

Figure 2. Mean proportions of correct reports of T2, given T1 correct ( $A B$ data), plotted as a function of lag and for each of the field groups. The solid line represents the static group and the dotted line represents the out group. Error bars represent standard errors of the mean. 
different task-irrelevant percept - that is, the illusion of visual motion, is equally able to attenuate the robust outcome known as the AB. However, what we are not yet able to show is whether the effect we find is obtainable by any motion, or whether it must be motion in a particular direction - that is, outward and away from the AB task. If, as Olivers and Nieuwenhuis argued, the background acts merely to trigger a more diffuse attentional state, it might be expected that motion toward the location of the AB task should attenuate the AB outcome equally well. We test this hypothesis in the next experiment.

\section{EXPERIMENT 2}

In order to test the idea that the direction of movement is critical to effect the outcome we reported above, we used the same starfield simulation technique, with the only change being that the dots now moved in the opposite direction to that in Experiment 1-that is, toward the primary AB task (see Figure 3).

\section{Method}

Participants. Eleven participants were recruited from the community panel, and each received $£ 5$ for a 45 -min session. The mean age of the participants was 29 years $(S D=4.8)$ and all had normal or corrected-to-normal visual acuity.

Stimuli and Procedure. We used the same stimuli and procedure from Experiment 1, except that now the round dots from the starfield moved from the periphery to the center of the screen, creating an inward motion. Dots disappearing near the RSVP stream were replaced by corresponding dots at their "origin" near the periphery. We will refer to this condition as in.

Design. A single factor, lag, with the same four levels as in Experiment 1 , was used in this experiment.

\section{Results and Discussion}

Overall T1 accuracy was $.89(S D=.09)$. As before, our analysis was based on correct T2 identification on trials for which T1 was also correct. Figure 4 shows the mean

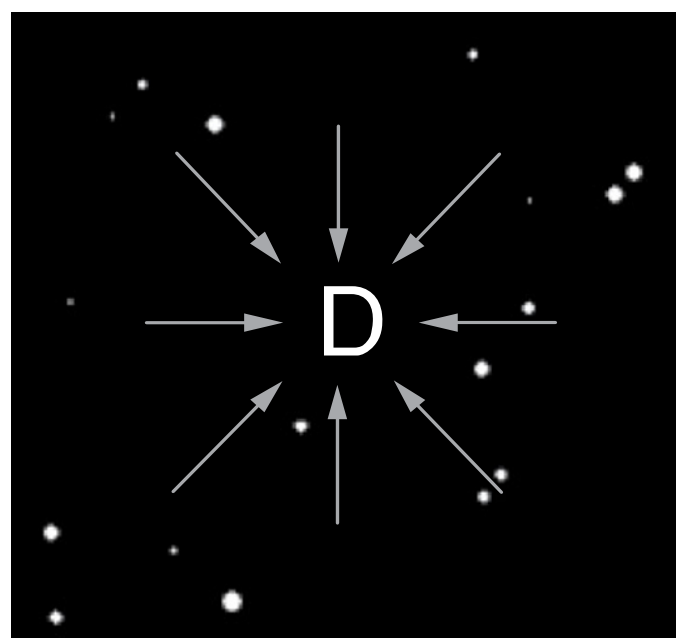

Figure 3. This example shows the in motion condition. The round dots move from the periphery to the center of the screen where the RSVP stream occurs. proportion of $\mathrm{AB}$ data plotted as a function of lag. Oneway ANOVA for all serial positions of T2 (lags) showed a significant effect $\left[F(3,30)=7.984, p<.05, \eta_{\mathrm{p}}^{2}=.444\right]$. Multiple comparisons using the Fisher's test showed that the main effect of lag was due to the difference between lag 2 and the two final lags (lag 6 and lag $8 ; p<.05$ ). No other comparisons reached significance.

The significant effect of lag indicates that an AB was found in this movement condition. However, a closer look at the data, comparing the present experiment with Experiment 1, reveals that participants' performance at lag 3 in the in condition was just $4 \%$ lower than in the out condition and $20 \%$ higher than in the static condition from Experiment 1. That is, the two movement conditions seemed to differ in the first lag, with the in condition revealing what appears to be a shorter duration $\mathrm{AB}$ than the static condition.

To statistically verify the above observation, we compared results from Experiments 1 and 2 by means of a two-way mixed ANOVA, with field as a between-subjects factor (3) and lag as a within-subjects factor (4). The main effect of field was statistically significant $[F(2,30)=$ $\left.3.427, p<.05, \eta_{\mathrm{p}}^{2}=.186\right]$, as was the main effect of lag $\left[F(3,90)=19.027, p<.05, \eta_{\mathrm{p}}^{2}=.388\right]$, with the interaction of field $\times \operatorname{lag}\left[F(6,90)=3.232, p<.05, \eta_{\mathrm{p}}^{2}=.177\right]$ significant as well. Multiple comparisons using Fisher's test revealed that at lag 2 , the out condition was significantly different from both the in and the static conditions, but the in and static conditions did not differ from each other. At lag 3, the two motion conditions did not differ from each other, but both were significantly different from the static condition.

This experiment was designed to test the hypothesis that task-irrelevant background motion, regardless of direction, is sufficient to attenuate the AB. The data instead support the conclusion that the direction of motion is important, with motion moving away from the primary task yielding the greatest degree of attenuation. Motion toward the primary task's location causes, at best, a significant, though short-duration AB effect, suggesting that the presence of apparent visual motion has some general property to reduce dual-task interference but that the effect is exacerbated by motion moving in a particular direction.

One issue from the previous experiments ${ }^{3}$ remains to be settled. Whereas we favor the account that background motion away from the locus of the RSVP stream, as in Experiment 1, produces less attention to-that is, draws attention away from - the target tasks, it is possible that the opposite in fact occurs. That is, as the background stars move outward, an illusion that the observer is actually moving toward the RSVP stream may have occurred (Gibson, 1979). Such an illusion might thus increase, rather than decrease, attention to the targets. Although the inward motion condition is a clue that the confounding illusion described above is likely not the cause of the effects we have observed, in the final experiment, we sought to determine whether background visual motion is required to obtain our effect, by creating a condition in which attention would be drawn to the periphery due to changes in the background, but not via any mechanism involving motion. 


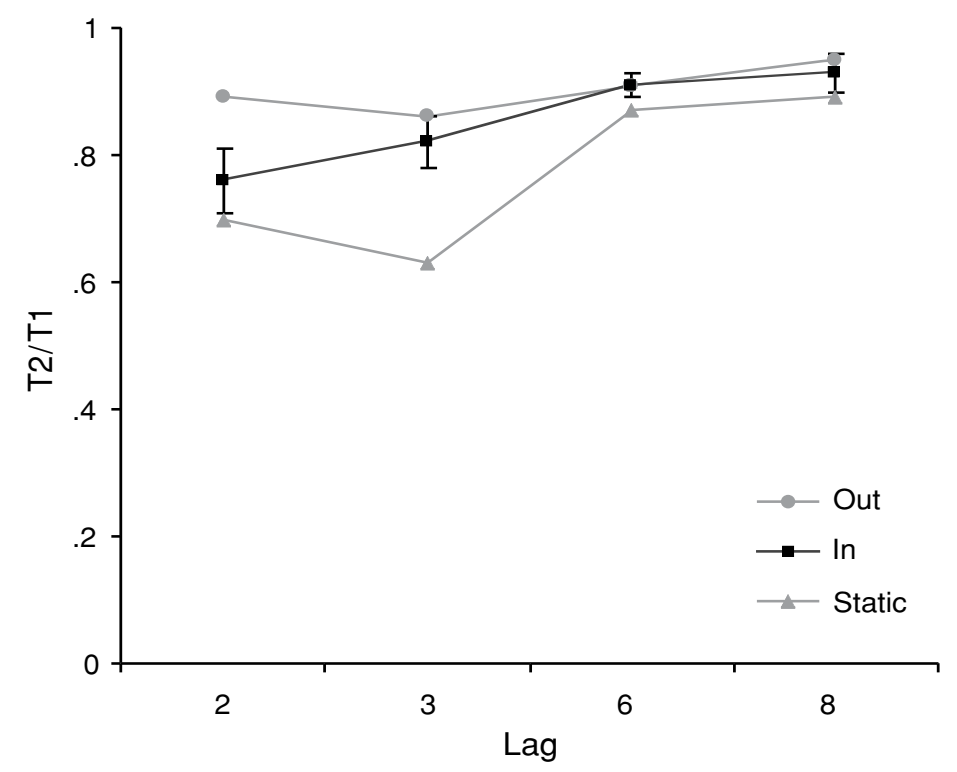

Figure 4. Mean proportions of correct $\mathrm{T} 2$, given a correct $\mathrm{T} 1$ response, plotted as a function of lag (AB data) for the in condition, are represented by the solid black line. Gray circles and triangles represent $A B$ data found in Experiment 1 for the out and static conditions, respectively. Error bars represent standard errors of the mean.

\section{EXPERIMENT 3}

In order to test the idea that the attenuation of the $\mathrm{AB}$ effect in previous experiments is not due to a target illusion created by motion of the background, we created a background designed to draw attention away from the target tasks but without the illusion of motion. Toward this end, we created a condition in which the same number of background dots that moved in Experiments 1 and 2 now flickered, as described below.

\section{Method}

Participants. Eleven participants were recruited from the community panel, and each received $£ 5$ for a 45 -min session. The mean age of the participants was 29 years $(S D=4.8)$ and all had normal or corrected-to-normal visual acuity.

Stimuli and Procedure. The method for the "flicker" experiment was the same as in the previous two experiments; the only difference was in the background field. In this experiment, the background starfield was created by repeatedly altering the contrast of the dots in the visual field, yielding the impression of "sparkling."

The sparkling starfield was created by randomly assigning positions to 50 "dots" inside a $700 \times 700$ pixel area surrounding the center of the screen. As in the previous two experiments, none of the dots could enter an area in the very center of the screen, which was reserved for the RSVP stream. Each of the dots could be either white or black (i.e., invisible), with a $50 \%$ probability. The sparkle effect was created by randomly reassigning the dots to be white or black every $160 \mathrm{msec}$. At any one time, only 25 dots were visible on the screen - a number very similar to the number of moving dots in Experiments 1 and 2.

Design. A single factor (lag) with the same four levels as in Experiment 1 was used in this experiment.

\section{Results and Discussion}

Overall T1 accuracy was $.90(S D=.06)$. We quantified the $\mathrm{AB}$ in the same way as in the two previous experiments, with correct $\mathrm{T} 2$ identification contingent on T1-correct trials. Figure 5 shows the mean proportion of AB data plotted as a function of lag. Repeated measures ANOVA for lag (4) revealed a significant $\mathrm{AB}[F(3,30)=6.835, p<$ $.05]$. Pairwise comparisons using Fisher's test revealed that lag 2 is significantly different from lag 6 and lag $8(p<$ $.05)$. No other comparison reached significance.

Although an AB effect was observed in the present condition, the important question is how the magnitude of this effect compares with the magnitude of the effect in previous conditions (Experiments 1 and 2), so that the question posed at the end of Experiment 2 can be answered. Toward this end, we performed an ANOVA on all conditions in a 4 (field) $\times 4$ (lag) design. An effect of field $[F(3,40)=$ $\left.2.84, p<.01, \eta_{\mathrm{p}}^{2}=.176\right]$ and $\operatorname{lag}[F(3,120)=24.97, p<$ $\left..01, \eta_{\mathrm{p}}^{2}=.384\right]$ was observed, as well as a marginally significant interaction $\left[F(9,20)=1.99, p=.066, \eta_{\mathrm{p}}^{2}=\right.$ .130]. Post hoc comparisons revealed the following outcomes: There were no significant differences among any of the four conditions at either lags 6 or 8 , suggesting that all conditions recovered baseline performance to the same extent at longer SOAs. At lag 2, the static (control) condition $(M=.69)$ differed from the out condition $(M=.88$, $p<.05)$ and marginally from the flicker condition $(M=$ $.82, p=.09$ ). None of the experimental conditions (out, in, flicker) differed from each other at this lag. At lag 3, the static condition was significantly different from the other 


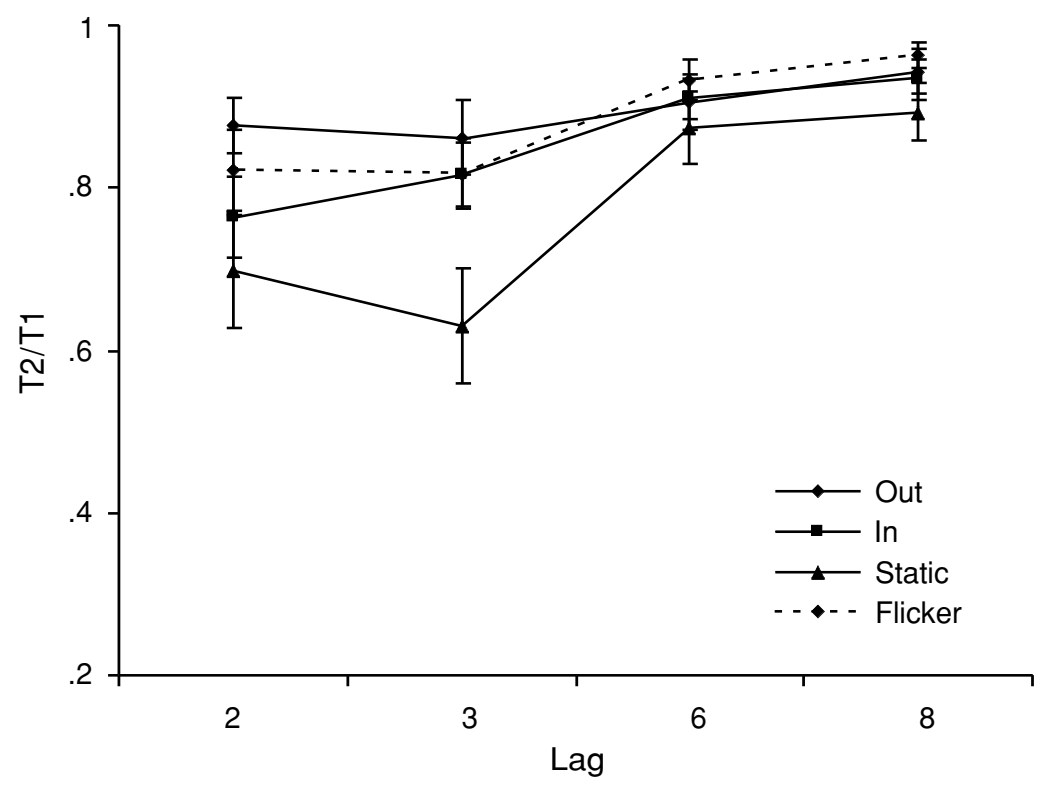

Figure 5. Mean proportions of correct $\mathrm{T} 2$, given a correct $\mathrm{T} 1$ response, plotted as a function of lag (AB data) for background conditions in all experiments. Black diamonds and triangles represent $A B$ data from Experiment 1 for the out and static conditions, respectively. Black squares represent data found in Experiment 2 for the in condition, and black circles represent data found in Experiment 3 for the flicker condition. Error bars represent standard errors of the mean.

three conditions: out $(M=.86, p<.05)$; in $(M=.81, p<$ $.05)$; flicker $(M=.82, p<.05)$. None of the latter three differed from each other.

The results of Experiment 3 enable us to address the important potential confound from Experiments 1 and 2: Does the attenuated $\mathrm{AB}$ we found-particularly in the outward motion condition - depend on a looming effect caused by a stationary target against a moving background? Given that we found no statistical difference between the out and flicker conditions - both yielding a significantly attenuated "blink" relative to the static condition-we can conclude that our effect likely results from attention being "withdrawn" from the RSVP stream. This conclusion is strengthened further by the result of the inward motion condition, in which, if a target receding illusion occurred, we should have found an increased AB; in fact, we found an attenuated effect.

\section{GENERAL DISCUSSION}

Our studies were motivated by the recent striking finding that the robust visual $\mathrm{AB}$ phenomenon is eliminated when participants listen to task-irrelevant auditory stimulation (Olivers \& Nieuwenhuis, 2005). The cross-modal source of the background stimulation, however, leaves unclear the extent to which such an outcome may be achieved by other means. As suggested by the authors, this effect may be mediated by increasing or decreasing the level of arousal, or by bringing participants to a positive mood state.

In response, our research examined whether the "state" considered by Olivers and Nieuwenhuis (2005) could also be triggered by manipulating the visual environment in a spatially specific manner, designed to attract attention away from the location of the RSVP stream containing the target tasks. The success of our manipulation was shown clearly in Experiment 1, in which the typically found robust $\mathrm{AB}$ effect was fully attenuated when stimuli in outward motion were used as a task-irrelevant background, in comparison with when the background did not imply motion. The possibility that the direction of motion could have a specific effect motivated Experiment 2, in which we found that motion going in the reverse direction - that is, from the periphery to the center of the screen-produced an $\mathrm{AB}$, albeit of smaller duration, in comparison with the nonmotion condition of Experiment 1. Finally, to be certain that the motion conditions of Experiments 1 and 2 did not induce an illusory effect on the target, per se, we conducted Experiment 3, in which attention was drawn away from the target tasks by a "flicker."

The results of these experiments enable the following conclusions to be drawn: (1) The presence of a taskirrelevant background implying motion attenuates the $\mathrm{AB}$, but the specific direction of motion (outward) may be more successful in attenuating the effect; (2) the background can emanate from the same modality as the $\mathrm{AB}$ 
task; and (3) the (outward) motion background most effective at attenuating the $\mathrm{AB}$ effect cannot be explained on the basis of target "looming," thereby ruling out that increased rather than decreased attention to the RSVP task lies at the root of our effect.

So what have we learned from the present research? First, we have found that background tasks qualitatively different from those used by Olivers and Nieuwenhuis (2005) can attenuate the AB. Second, and consistent with our hypothesis, there may be a visual spatial component mediating our effect and possibly Olivers and Nieuwenhuis's as well. Specifically, we suggest that the outward motion and flicker conditions place attention in a different spatial location - that is, farther away from the AB taskthan where it is normally allocated when no attentionally demanding background is present (in other words, the typical $\mathrm{AB}$ condition). In turn, this prevents the overallocation of attention to the first target task, leaving resources available for $\mathrm{T} 2$. The music manipulation by Olivers and Nieuwenhuis, similarly, may have moved attention away from the location of the AB task. Relevant to this hypothesis, Shapiro and his colleagues revealed that, whereas attention is normally allocated to the center of an attended visual field, arousal and anxiety redirect attention to the visual periphery (Shapiro \& Johnson, 1987; Shapiro \& Lim, 1989). Perhaps our background visual motion and flicker conditions, as well as Olivers and Nieuwenhuis's music and subjects' "thoughts of their holidays," may have their effects mediated via arousal, as suggested by Olivers and Nieuwenhuis. We hasten to point out that such a notion is not inconsistent with the conclusion drawn by Snodgrass et al. (1993) that relaxation has a beneficial effect on attentionally demanding tasks. Thus, somewhat paradoxically, both arousal and relaxation may serve the same end of reducing attention to the spatial location of the AB task. Finally, the partial attenuation of the full AB effect, witnessed in the inward motion condition (Experiment 2), can be argued to be the result of the presence of (any) motion removing some attention from the location of the AB task, though to a lesser extent than when motion is in the opposite direction and can exert a maximal effect.

Consistent with our hypothesis that the present (effective) background conditions cause a decreased attentional allocation to the first target, Crebolder and Ostaniewicz (2001) found that, when participants were presented with a three-target $\mathrm{AB}$ task, there was a significantly reduced $\mathrm{AB}$ to the second target, even though this second target, when presented as part of a traditional two-target $\mathrm{AB}$ task, revealed the typical AB outcome. Perhaps the knowledge of the third target task motivated participants to reserve attentional allocation to the first target, with an outcome consistent with that found in the present report.

Further research needs to be undertaken to determine the validity of this argument and to elucidate more precisely the behavioral conditions under which this striking reduction in magnitude of the $\mathrm{AB}$ outcome is manifested. This is not to say that any diffuse attentional state should be expected to effect the present outcome. Such states arising from, for example, patients with frontal lobe damage or a developmentally immature brain, might yield an underallocation of attention required for successful performance, in the latter case, or a failure to establish an appropriate target template required for successful performance in a task such as the $\mathrm{AB}$, in the former case. Toward a better understanding of the role of attention in dual tasks such as the $\mathrm{AB}$, physiological indices - for example, the ERP P300 component, shown to be sensitive to the allocation of attention (Arnell, Helion, Hurdelbrink, \& Pasieka, 2004) - could profitably lend themselves to an understanding of the underlying mechanism by which this effect occurs. In support of this contention, a recent report by Kessler et al. (2005) revealed an attenuated M300 (MEG equivalent of the P300) response to a masked versus unmasked first target in an AB paradigm. Kessler and colleagues argue that the presence of the mask, required to produce the $\mathrm{AB}$ outcome, forces $\mathrm{T} 1$ processing into a robust state that is difficult to perturb; until such perturbation occurs, T2 processing is delayed. Perhaps the presence of a potent background stimulus, such as that employed by the present investigators as well as by Olivers and Nieuwenhuis, removes attentional resources, in turn causing the T1 mask to be less likely to cause such a perturbed state. Finally, in support of the notion that first and second target tasks compete for attentional resources, in a recent report, Shapiro, Schmitz, Martens, Hommel, and Schnitzler (2006) showed a positive correlation between the magnitude of the M300 to the first target and the magnitude of the resulting $\mathrm{AB}$ - that is, larger T1 M300 potentials were associated with a larger $\mathrm{AB}$.

\section{REFERENCES}

Arnell, K. M., Helion, A. M., Hurdelbrink, J. A., \& Pasieka, B. (2004). Dissociating sources of dual-task interference using human electrophysiology. Psychonomic Bulletin \& Review, 11, 77-83.

Brainard, D. H. (1997). The Psychophysics Toolbox. Spatial Vision, 10, 433-436.

Chun, M. M., \& Potter, M. C. (1995). A two-stage model for multiple target detection in rapid serial visual presentation. Journal of Experimental Psychology: Human Perception \& Performance, 21, 109-127.

Crebolder, J. M., \& Ostaniewicz, A. J. (2001). Spatial and temporal factors in the attentional blink. Poster presented at the 42nd Annual Meeting of the Psychonomic Society, Orlando, Florida.

Gibson, J. J. (1979). The ecological approach to visual perception. Chapter 14: The theory of information pickup and its consequences (pp. 238-263). Boston: Houghton Miflin.

Giesbrecht, B., \& Di Lollo, V. (1998). Beyond the attentional blink: Visual masking by object substitution. Journal of Experimental Psychology: Human Perception \& Performance, 24, 1454-1466.

Joseph, J. S., Chun, M. M., \& NaKayama, K. (1997). Attentional requirements in a "preattentive" feature search task. Nature, 379, 805807.

Kellie, F. J., \& ShapIRo, K. L. (2004). Object file continuity predicts attentional blink magnitude. Perception \& Psychophysics, 66, 692712.

Kessler, K., Schmitz, F., Gross, J., Hommel, B., Shapiro, K., \& SCHNitzler, A. (2005). Target consolidation under high temporal processing demands as revealed by MEG. NeuroImage, 26, 10301041.

OgaWa, T., \& SUzUKI, N. (2004). On the saliency of negative stimuli: Evidence from the attentional blink. Japanese Psychological Research, 46, 20-30.

Olivers, C. N. L., \& Nieuwenhuis, S. (2005). The beneficial effect 
of concurrent task-irrelevant mental activity on temporal attention. Psychological Science, 16, 265-269.

Olson, I. R., Chun, M. M., \& Anderson, A. K. (2001). Effects of phonological length on the attentional blink for words. Journal of Experimental Psychology: Human Perception \& Performance, 27, 1116-1123.

Pelli, D. G. (1997). The VideoToolbox software for visual psychophysics: Transforming numbers into movies. Spatial Vision, 10, 437-442.

Raymond, J. E., Shapiro, K. L., \& Arnell, K. M. (1992). Temporary suppression of visual processing in an RSVP task: An attentional blink? Journal of Experimental Psychology: Human Perception \& Performance, 18, 849-860.

Ross, N. E., \& Joliccur, P. (1999). Attentional blink for color. Journal of Experimental Psychology: Human Perception \& Performance, 25, 1483-1494.

Shapiro, K. L., Arnell, K. M., \& Raymond, J. E. (1997). The attentional blink. Trends in Cognitive Sciences, 1, 291-296.

Shapiro, K. L., Caldwell, J., \& Sorensen, R. E. (1997). Personal names and the attentional blink: The "cocktail party" effect. Journal of Experimental Psychology: Human Perception \& Performance, 23, 504-514.

Shapiro, K. L., \& Johnson, T. L. (1987). Effects of arousal on attention to central and peripheral visual stimuli. Acta Psychologica, 66, $157-172$.

Shapiro, K. L., \& LiM, A. (1989). The impact of anxiety on visual attention to central and peripheral events. Behaviour Research \& Therapy, 27, 345-351.

Shapiro, K. L., Raymond, J. E., \& Arnell, K. M. (1994). Attention to visual pattern information produces the attentional blink in rapid se- rial visual presentation. Journal of Experimental Psychology: Human Perception \& Performance, 20, 357-371.

Shapiro, K. L., Schmitz, F., Martens, S., Hommel, B., \& SchnitzLER, A. (2006). Resource sharing in the attentional blink. NeuroReport, 17, 163-166.

Sheppard, D. M., Duncan, J., Shapiro, K. L., \& Hillstrom, A. P. (2002). Objects and events in the attentional blink. Psychological Science, 13, 410-415.

Smilek, D., Enns, J. T., Eastwood, J. D., \& Merikle, P. M. (in press). Relax! Cognitive strategy influences visual search. Visual Cognition.

Snodgrass, M., Shevrin, H., \& KopKa, M. (1993). The mediation of intentional judgments by unconscious perceptions: The influences of task strategy, task preference, word meaning, and motivation. Consciousness \& Cognition, 2, 169-193.

\section{NOTES}

1. The gap-duration task demanded that participants judge the temporal interval between two successive letter stimuli in the RSVP stream. This experiment successfully replicates the one reported by Shapiro, Raymond, and Arnell (1994, Experiment 5A).

2. In their experiment, Chinese ideographs (Kanji) were rated according to their pleasantness as being positive, negative, or neutral.

3. Our thanks to the action editor for pointing out this potential confound.

(Manuscript received Februrary 21, 2005; revision accepted for publication December 19, 2005.) 